\title{
Embedded Cell Model of Three-Dimensional Two-Phase Composites
}

\author{
S.G. Zhan, T.C. Wang and B.H. Ji \\ LNM, Institute of Mechanics, Chinese Academy of Sciences, 15 Zhong Guan Cun Road, \\ Beijing 100080, P.R. China
}

Keywords: Embedded Cell Model, Three-Dimensional, Two-Phase Composites

\begin{abstract}
An embedded cell model is presented to obtain the effective elastic moduli and the elastic-plastic stress-strain relations of three-dimensional two-phase particulate composites. Each cell consists of an ellipsoidal inclusion surrounded by a finite ellipsoidal matrix that embedded in an infinite matrix. When both matrix and particle are elastic, the effective elastic moduli are derived which is an exact analytic formula without any simplified approximation that can be expressed in an explicit form. Further, the elastic-plastic stress-strain relations are obtained for spherical cells and oblate spheroid cells, in which the matrix is elastic and the particle is elastic-plastic. In addition, the macroscopic elastic-plastic constitutive relation of particle reinforced composites (PRC) is investigated by a systematic approach [1] in which the matrix is elastic-plastic and the particle is elastic.
\end{abstract}

\section{INTROUCTION}

The recent rapid progress in materials science has led to the production of advanced composites with superior mechanical properties and gives rise to the intensive study on predicting the behavior of heterogeneous materials. One of the most basic problems in composite-materials theory is the prediction of effective elastic properties of composites which is the subject of many investigations. Equivalent-inclusion principle was presented by Eshelby[2] in 1957. There are several micromechanics models to estimate the effective moduli of composites[3-8]. The closed-form interacting solutions for the overall elastic moduli of an isotropic matrix with various multi-phase and multi-shape isotropic inclusions are derived by Zheng and $\mathrm{Du}[9]$.

Qiu and Weng[10] developed an average method to predict the nonlinear constitutive relation of composite materials based on the modified Mori and Tanaka procedure. An alternative approach proposed by Duva and Hutchinson[11] is based on the solution of a kernel problem, in which an isolated inclusion is embedded in an infinite matrix of nonlinear material. Zhu and Zbib[12] investigated the mechanical properties of composites based on a finite unit cell model accounting for the interaction of particles in composites with periodic microstructures (e.g. Llorca and González[13]). Each unit cell consists of a rigid inclusion surrounded by a plastically deforming material.

For brevity, the symbolic notation will be used in the general theory in this paper. Bold-face, Greek letters denote the 2nd-rank tensors, and ordinary capital letters denote the 4th-rank ones. The inner product of two tensors is written such that $\sigma \varepsilon=\sigma_{i j} \varepsilon_{i j}, \mathbf{L} \varepsilon=L_{i j k l} \varepsilon_{k 1}$, and $\mathbf{L S}=\mathrm{L}_{\mathrm{ijk} l} \mathrm{~S}_{\mathrm{klmn}}$, in terms of the indicial components. 


\section{EMBEDDED CELL MODEL AND BASIC FORMULAE}

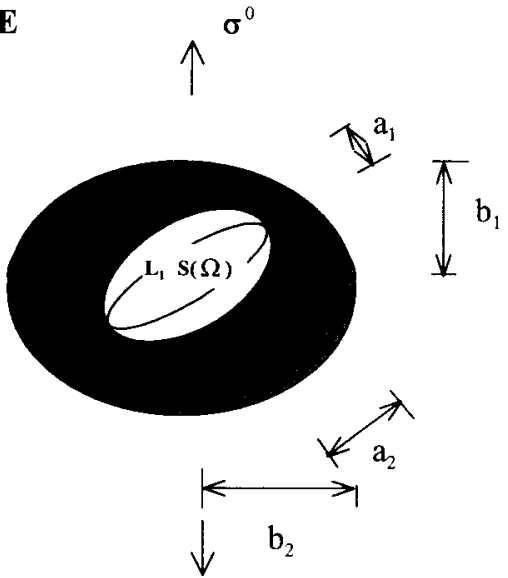

An ellipsoidal elastic inclusion $\Omega$ is considered in a homogeneous infinite elastic body. The traction at infinity is prescribed to correspond to uniform stresses. An embedded cell model consists of an ellipsoidal inclusion $\Omega$ surrounded by a finite eilipsoid $\mathrm{V}$ and is embedded in an infinite matrix (Fig. 1). The embedded cell model is different from the classical three-phase and two-phase model, because the embedded cell is only concerned in matrix phase and inclusion phase and it is embedded in an infinite matrix so that the traction and the displacements of the cell's boundary are determined by the uniform remote loads. The elasticity tensors of the matrix and the ellipsoidal inclusion $\Omega$ are $\mathbf{L}_{0}$ and $\mathbf{L}_{1}$, respectively. (see Fig. 1).

\subsection{Effective Elastic Moduli}

Fig. 1 The embedded cell model

Suppose that the infinite homogeneous elastic body is subjected to farfield stress $\sigma^{0}$, with accompanying strain $\varepsilon^{0}$. The strain and stress fields in the matrix naturally differ from $\sigma^{0}$ and $\varepsilon^{0}$ due to the presence of the inclusion. Denoting these disturbances by $\sigma$ and $\varepsilon$ respectively, the total stress and strain are given by

$$
\sigma_{\text {total }}=\sigma^{0}+\sigma, \quad \varepsilon_{\text {total }}=\varepsilon^{0}+\varepsilon
$$

By means of Eshelby's solutions and the equivalent eigenstrain principle [14], the stress and strain in the inclusion are uniform, given by

$$
\int_{\Omega} \sigma \mathrm{dV}=\Omega \sigma=\Omega \mathbf{L}_{0}\left(\boldsymbol{\varepsilon}-\boldsymbol{\varepsilon}^{*}\right)=\Omega \mathbf{L}_{0}(\mathbf{S}(\Omega)-\mathbf{I}) \varepsilon^{*}, \quad \int_{\Omega} \varepsilon \mathrm{dV}=\Omega \varepsilon=\Omega \mathbf{S}(\Omega) \varepsilon^{*}
$$

where eigenstrain $\varepsilon^{*}$ can be expressed as follows

$$
\varepsilon^{*}=-\left[\mathbf{L}_{0}+\left(\mathbf{L}_{1}-\mathbf{L}_{0}\right) \mathbf{S}(\Omega)\right]^{-1}\left[\mathbf{L}_{1}-\mathbf{L}_{0}\right] \varepsilon^{0}=-\mathbf{A} \varepsilon^{0}
$$

here $\mathbf{A}$ is the concentration factor tensor, $A=\left[\mathbf{L}_{0}+\left(\mathbf{L}_{1}-\mathbf{L}_{0}\right) \mathbf{S}(\Omega)\right]^{-1}\left[\mathbf{L}_{1}-\mathbf{L}_{0}\right]$

and $\mathbf{S}(\Omega)$ is Eshelby's tensor for the ellipsoid $\Omega$. The components of the S-tensor for a general isotropic spheroid are given in Appendix.

In terms of Tanaka-Mori's theorem [14,15], we have

$$
\int_{-\Omega} \sigma \mathrm{dV}=\Omega \mathbf{L}_{0}(\mathbf{S}(\mathrm{V})-\mathrm{S}(\Omega)) \varepsilon^{*}, \quad \int_{-\Omega} \varepsilon \mathrm{dV}==\Omega(\mathbf{S}(\mathrm{V})-\mathrm{S}(\Omega)) \varepsilon^{*}
$$

where $S(V)$ is Eshelby's tensor for the ellipsoid V. Eq. (4) show the volume integrals of the stress $\sigma$ and the strain $\varepsilon$ over $V-\Omega$ are independent of the absolute position of $V$ and $\Omega$; they depend only on the Eshelby's tensors of $\mathrm{V}$ and $\Omega$; they vanish when $\mathrm{V}$ and $\Omega$ are similar in shape and have the same orientation.

Then, the average stress and strain of the embedded cell are

$$
\overline{\boldsymbol{\sigma}}=\frac{1}{\mathrm{~V}} \int_{\mathrm{V}} \boldsymbol{\sigma}_{\text {total }} \mathrm{dV}=\mathbf{L}_{0}[\mathbf{I}+\rho(\mathbf{I}-\mathbf{S}(\mathrm{V})) \mathbf{A}] \mathbf{\varepsilon}^{0}, \quad \overline{\boldsymbol{\varepsilon}}=\frac{1}{\mathrm{~V}} \int_{\mathrm{V}} \boldsymbol{\varepsilon}_{\text {tota }} \mathrm{dV}=[\mathbf{I}-\rho \mathbf{S}(\mathrm{V}) \mathbf{A}] \boldsymbol{\varepsilon}^{0}
$$

where $\rho$ is the volume fraction of the inclusion, $\rho=\Omega / \mathrm{V}$.

The effective elastic moduli of the cell, $\mathbf{L}$, then follow from $\bar{\sigma}=\mathbf{L} \bar{\varepsilon}$, as

$$
\mathbf{L}=\mathbf{L}_{0}\left\{\mathbf{I}+\rho \mathbf{A}[\mathbf{I}-\rho \mathbf{S}(\mathbf{V}) \mathbf{A}]^{-1}\right\}
$$

Expression (6) is an exact analytic formula for the effective elastic moduli of the embedded cell model. In equation (6), $\mathbf{S}(\mathrm{V})$ reflects the effect of the shape of the ellipsoid $\mathrm{V}$, and $\mathbf{S}(\Omega)$ is relative to the shape of the inclusion $\Omega$. So, the explicit expression (6) is simple but quite meaningful.

When the ellipsoid $\mathrm{V}$ and the ellipsoidal inclusion $\Omega$ are similar in shape and have the same 
orientation, substituting $\mathbf{S}(\mathrm{V})=\mathbf{S}(\Omega)=\mathbf{S}$ into Eq. (6), we find that the effective elastic moduli are the same as those obtained by Mori-Tanaka method[16].

When the inclusion is spherical, two aspect ratios of the ellipsoid $\mathrm{V}$ are considered in order to investigate the effect of the shape of the cell on the effective elastic moduli. For the spherical $\mathrm{V}$, $\mathrm{S}(\mathrm{V})(=\mathrm{S}(\Omega))$ is given in A3. Eq. (6) readily provides the effective bulk and shear moduli of the cell which are the same as those by the Mori-Tanaka method [8] and displayed in Fig. 2. For the rotative

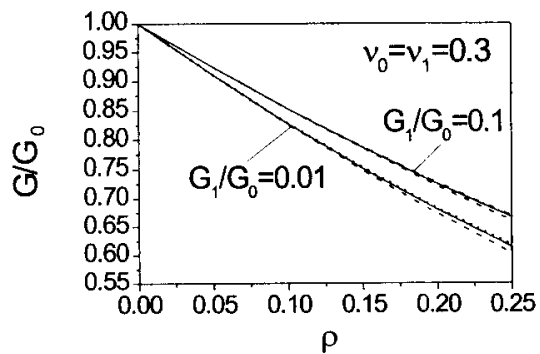

(a) $G_{1} / G_{0}<1$

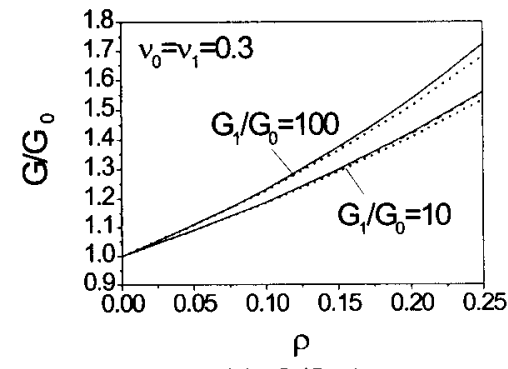

(b) $G / G_{0}>1$

Fig. 2 Normalized effective shear moduli vs volume fraction

..... Mori-Tanaka method [8]; - - -Generalized self-consistent method [5];

- Spherical inclusion in oblate ellipsoidal matrix

oblate spheroid V, $\mathbf{S}(\mathrm{V})$ is given (in Appendix A1) for the aspect ratio $\alpha_{0}=b_{1} / b_{2}=1 / 2$, assuming direction 1 to be the axis of rotation. Substituting $S(V)$ and $S(\Omega)$ into Eq.(6), the effective moduli are obtained as showed in Fig. 2, along with the solutions of other micromechanics models. As shown in these figures, the moduli are close to the solutions from the generalized self-consistent model[5] and the Mori-Tanaka method[8], which indicates that the effect of the shape of the cell on the effective elastic moduli is quite small.

\subsection{Elastic-Plastic Stress-Strain Relations}

The embedded cell model is developed to obtain the elastic-plastic stress-strain relations of twophase particulate composites, in which the matrix is elastic and the particle is elastic-plastic. The macroscopic elastic-plastic constitutive relation of the cell is expressed by $\bar{\sigma}=\mathbf{L}_{\text {eff }} \bar{\varepsilon}$. The effective moduli $\mathbf{L}_{\text {eff }}$ can be obtained based on the formulae in the above section, in which the elastic moduli of the particle $E_{1}$ and $v_{1}$ are replaced by the secant moduli of the particle $E_{1}^{s}$ and $v_{1}^{s}$.

When the effective strain of the particle is given, the effective stress can be obtained as based on the power-law equation

$$
\sigma_{\mathrm{e}}=\mathrm{h}\left(\varepsilon_{\mathrm{e}}\right)^{\mathrm{n}}
$$

According to deformation theory of plasticity, the deviatoric stress is

The secant moduli of the particle are

$$
\sigma_{i j}^{\prime}=\left(\frac{2}{3} \frac{\sigma_{e}}{\varepsilon_{e}}\right) \varepsilon_{i j}^{\prime}
$$

$$
\left(\mathrm{L}_{\mathrm{l}}^{\mathrm{s}}\right)_{\mathrm{ijk} \mathrm{k}}=\frac{1}{3} \frac{\sigma_{\mathrm{e}}}{\varepsilon_{\mathrm{e}}}\left(\delta_{\mathrm{ik}} \delta_{\mathrm{jl}}+\delta_{\mathrm{jk}} \delta_{\mathrm{il}}\right)+\left(\mathrm{K}_{\mathrm{l}}-\frac{2}{9} \frac{\sigma_{\mathrm{e}}}{\varepsilon_{\mathrm{e}}}\right) \delta_{\mathrm{ij}} \delta_{\mathrm{kl}}
$$

Then, the elastic moduli of the cell can be expressed by

$$
\mathbf{L}_{\mathrm{eff}}=\mathbf{L}_{0}\left\{\mathbf{I}+\rho \mathbf{A}_{\mathbf{s}}\left[\mathbf{I}-\rho \mathbf{S}(\mathrm{V}) \mathbf{A}_{\mathrm{s}}\right]^{-1}\right\}
$$

here $\mathbf{A}_{\mathbf{s}}=\left[\mathbf{L}_{0}+\left(\mathbf{L}_{1}^{\mathbf{s}}-\mathbf{L}_{0}\right) \mathbf{S}(\Omega)\right]^{-1}\left[\mathbf{L}_{1}^{\mathbf{s}}-\mathbf{L}_{0}\right]$.

Since the particle's strain is 


$$
\boldsymbol{\varepsilon}=\left\{\mathbf{I}-\mathbf{S}(\Omega)\left[\mathbf{L}_{0}+\left(\mathbf{L}_{1}^{\mathbf{s}}-\mathbf{L}_{0}\right) \mathbf{S}(\Omega)\right]^{-1}\left[\mathbf{L}_{1}^{\mathbf{s}}-\mathbf{L}_{0}\right]\right\} \varepsilon^{0}=\left[\mathbf{I}-\mathbf{S}(\Omega) \mathbf{A}_{\mathbf{s}}\right] \boldsymbol{\varepsilon}^{0}=\mathbf{B}_{\mathbf{s}} \varepsilon^{0}
$$

then, the average stress and strain of the cell can be calculated

$$
\begin{gathered}
\overline{\boldsymbol{\sigma}}=\mathbf{L}_{0}\left[\mathbf{I}+\rho(\mathbf{I}-\mathbf{S}(\mathrm{V})) \mathbf{A}_{\mathbf{s}}\right] \boldsymbol{\varepsilon}^{0}=\mathbf{L}_{0}\left[\mathbf{I}+\rho(\mathbf{I}-\mathbf{S}(\mathrm{V})) \mathbf{A}_{\mathbf{s}}\right]\left(\mathbf{B}_{\mathbf{s}}\right)^{-1} \boldsymbol{\varepsilon}=\overline{\mathbf{L}} \boldsymbol{\varepsilon} \\
\overline{\boldsymbol{\varepsilon}}=\left[\mathbf{I}-\rho \mathbf{S}(\mathbf{V}) \mathbf{A}_{\mathbf{s}}\right] \boldsymbol{\varepsilon}^{0}=\mathbf{C}_{\mathbf{s}}\left(\mathbf{B}_{\mathbf{s}}\right)^{-1} \boldsymbol{\varepsilon}
\end{gathered}
$$

where $\overline{\mathbf{L}}=\mathbf{L}_{0}\left[\mathbf{I}+\rho(\mathbf{I}-\mathbf{S}(\mathrm{V})) \mathbf{A}_{\mathbf{s}}\right]\left(\mathbf{B}_{\mathbf{s}}\right)^{-1}$

It is easily seen that the stress and strain relations of the cell can be obtained exactly and in closed form once the strain of the particle is given.

In this paper, the elastic-plastic relation curves of the composite are calculated for spherical cell and oblate spheroid cell (the aspect ratio is 0.5 ). The ellipsoid V is similar to and coaxial with particle $\Omega$ and the volume fraction $\rho$ of the particle is 0.5 . Three materials are considered. For the uniaxial stress state, suppose that direction 1 is the loading axis, according to the deviatoric strain of the particle $\left(\varepsilon_{22}^{\prime}=\varepsilon_{33}^{\prime}=-\varepsilon_{11}^{\prime} / 2\right)$ and the effective strain $\varepsilon_{\mathrm{e}}$ of the particle, we have

$$
\varepsilon_{\mathrm{e}}=\varepsilon_{11}^{\prime}
$$

When the effective strain of the particle is given, $\overline{\mathbf{L}}$ can be obtained in terms of Eq. (14). Because the average stresses $\bar{\sigma}_{22}$ and $\bar{\sigma}_{33}$ of the cell are equal to zero, the average strain (hydrostatic strain) $\varepsilon_{\mathrm{m}}$ of the particle can be calculated by means of Eqs. (12) and (15). Then the stress and strain relations of the cell are obtained which are displayed in Fig. 3. As shown in figures, the effect of the shapes of the cells on the marcostress and macrostrain relation curves of the cell is little. Although the particle is power hardening material, the nonlinear properties of the cell are slight because the matrix is elastic material. The effect of the elastic moduli of the particle on the stress and strain relations is not significant in uniaxial stress state.
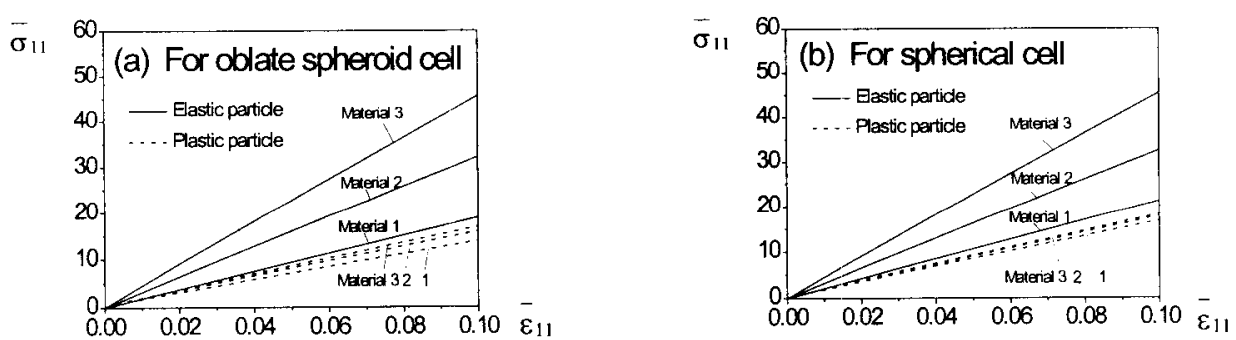

Fig. 3 The stress-strain curves in uniaxial stress state

Material 1: $\mathrm{E}_{0}=450 \mathrm{GPa}, v_{0}=0.17, \mathrm{E}_{1}=70 \mathrm{GPa}, \quad v_{1}=0.33, \mathrm{~h}=700 \mathrm{MPa}$ and $\mathrm{n}=0.1$
Material 2: $\mathrm{E}_{0}=450 \mathrm{GPa}, v_{0}=0.17, \mathrm{E}_{1}=225 \mathrm{GPa}, v_{1}=0.33, \mathrm{~h}=700 \mathrm{MPa}$ and $\mathrm{n}=0.1$
Material 3: $\mathrm{E}_{0}=450 \mathrm{Gpa}, v_{0}=0.17, \mathrm{E}_{1}=450 \mathrm{GPa}, v_{1}=0.33, \mathrm{~h}=700 \mathrm{MPa}$ and $\mathrm{n}=0.1$

\section{ELASTIC-PLASTIC CONSTITUTIVE RELATION OF PARTICLE REINFORCED METAL-MATRIX COMPOSITE}

The particle reinforced metal-matrix composite was idealized as uniformly distributed periodic arrays of aligned unit spherical cells, and each cell consists of an elastic spherical inclusion surrounded by an elastic-plastically deforming matrix.

Generally, we assume that the macro elastic-plastic constitutive relationship can be expressed with deformation theory as follows,

$$
E_{i j}=E_{i j}^{e}+E_{i j}^{p}=C_{i j k l} \Sigma_{k l}+\frac{3}{2} \frac{E_{e}^{p}}{A\left(E_{e}^{p}\right)} S_{i j}
$$

where $E_{i j}, E_{i j}^{e}, E_{i j}^{p}$ are the total macro strain, elastic strain and plastic strain, respectively, $C_{i j k l}$ is the 
macro elastic compliance tensor of the unit cell, $\Sigma_{\mathrm{kl}}$ is the total macro stress, $\mathrm{S}_{\mathrm{ij}}$ is the deviatoric part of average macrostress.

In Eq.(16), the function $A\left(E_{e}^{p}\right)$ is describing the work hardening properties of the unit cell composites. It is worth to noting that the constitutive relationship of the composites will be completely determined if the function $A\left(E_{e}^{p}\right)$ is given. To simplify the evaluation of the function $A\left(E_{e}^{p}\right)$, the elastic deformations of both inclusion and matrix were neglected, then the particle can be simplified as a rigid inclusion, and the matrix was simplified as an incompressible power law material. The macro elastic compliance $\mathrm{C}_{\mathrm{ijk} \mathbf{k}}$ can be obtained according to Mori and Tanaka Method. In the calculation, the displacement function can be expanded in series with unknown coefficients which can be determined by the minimum principle (see Ji and Wang[1]).

The macro stress and strain relation of unit cell is obtained,

$$
\frac{\Sigma_{\mathrm{e}}}{\sigma_{0}}=\frac{\Sigma_{\mathrm{n}}}{\sigma_{0}}\left(\frac{\mathrm{E}_{\mathrm{e}}}{\alpha \varepsilon_{0}}\right)^{\mathrm{n}}
$$

where

$$
\frac{\Sigma_{\mathrm{n}}}{\sigma_{0}}=\mathrm{F}(\mathrm{f}, \mathrm{n}, \mathrm{H} / \mathrm{R})=\left(\frac{3}{2}\right)^{\mathrm{n}+1} \frac{1}{\mathrm{~V}} \int_{\mathrm{V}_{\mathrm{a}}} \hat{\varepsilon}_{\mathrm{e}}^{\mathrm{n}+1} \mathrm{dV} \overline{\mathrm{E}}^{\mathrm{n}}
$$

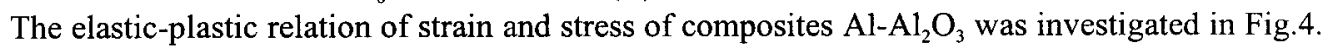
The predicted results were compared with the finite element results of Llorca and González[13]. The relation of hardening factor $F(\rho, n)$ versus particle volume fraction $\rho$ was calculated at a various value of strain hardening exponent $n$, the calculating results were described in Fig.5

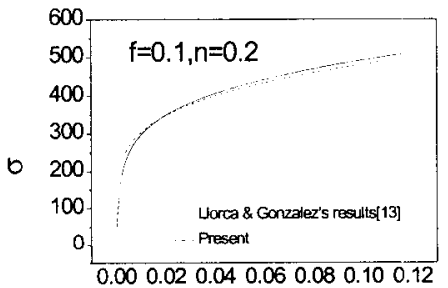

$\varepsilon$

Fig. 4 Predicted stress and elasticplastic strain curve compared with FEM results of Llorca and González[13]

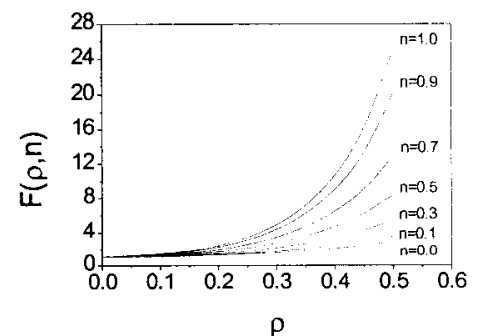

Fig. 5 Effects of strain hardening exponent and particle volume fraction on the constitutive relation of composites

\section{CONCLUSION}

An embedded cell model is presented to obtain the effective elastic moduli for three-dimensional two-phase composites which is an exact analytic formula without any simplified approximation and can be expressed in an explicit form. For composites with the spherical particles, the present results are close to those of the solutions from the generalized self-consistent model and the Mori-Tanaka method. The elastic-plastic stress-strain relations of two-phase particulate composites are obtained by the embedded cell model, in which the matrix is elastic and the particle is elastic-plastic. In addition, the macroscopic elastic-plastic constitutive relation of particle reinforced composites (PRC) is investigated by a systematic approach[1] in which the matrix is elastic-plastic and the particle is elastic.

Acknowledgement The research presented here was supported by the National Natural Science Foundation of China (No. 19704100) and the National Natural Science Foundation of Chinese Academy of Sciences (Project KJ951-1-201). 


\section{REFERENCES}

1. B. H. Ji and T. C. Wang, Acta Mech. Sinica, 15 (1999) 344-354.

2. J. D. Eshelby, Proceedings of the Royal Society, London, Series A, 240 (1957) 367-396.

3. R. Hill, J. Mech. Phys. Solids, 13 (1965) 213-222.

4. B. Budiansky, J. Mech. Phys. Solids, 13 (1965) 223-227.

5. R. M. Christensen and K. H. Lo, J. Mech. Phys. Solids, 27 (1979) 315-330.

6. R. Mclaughlin, Int. J. Engng Sci., 15 (1977) 237-244.

7. T. Mori and K. Tanaka, Acta Metall., 21 (1973) 571-574.

8. G. J. Weng, Int. J. Eng. Sci., 22 (1984) 845-856.

9. Q. S. Zheng and D. X. Du, Key Engineering Materials, 145-149 (1998) 479-488.

10. Y. P. Qiu and G. J. Weng, Int. J. Solids Struct., 27 (1991) 1537-1550.

11. J. M. Duva and J. W. Hutchinson, Mech. Mater., 3 (1984) 41-54.

12. H. T. Zhu and H. M. Zbib, Int. J. Plasticity, 11 (1995) 471-499.

13. J. Llorca and C. González, J. Mech. Phys. Solids, 40 (1998) 1-28.

14. T. Mura, Micromechanics of defects in solids. Martinus Nijhoff, The Hague, 1982.

15. K. Tanaka and T. Mori, J. Elasticity, 2 (1972) 199-200.

16. Y. H. Zhao, G. P. Tandon and G. J. Weng. Acta Mechanica, 76 (1989) 105-131.

\section{Appendix Components of Eshelby's $S_{i j k k}$ Tensor}

For a spheroidal inclusion with the symmetric identified as $x_{l}$, the components of Eshelby's tensor $S_{i j k l}$ are:

$$
\begin{aligned}
& \mathrm{S}_{1111}=\frac{1}{2\left(1-v_{0}\right)}\left\{1-2 v_{0}+\frac{3 \lambda^{2}-1}{\lambda^{2}-1}-\left[1-2 v_{0}+\frac{3 \lambda^{2}}{\lambda^{2}-1}\right] \mathrm{g}\right\} \\
& \mathrm{S}_{2222}=\mathrm{S}_{3333}=\frac{1}{8\left(1-v_{0}\right)} \lambda^{2}-1 \\
& \lambda^{2}-1 \\
& \mathrm{~S}_{2233}=\mathrm{S}_{3322}=\frac{1}{4\left(1-v_{0}\right)}\left[1-2 v_{0}-\frac{9}{4\left(\lambda^{2}-1\right)}\right] \mathrm{g} \\
& \left.\mathrm{S}_{2211}=\mathrm{S}_{3311}=-\frac{\lambda^{2}}{2\left(\lambda^{2}-1\right)}-\left[1-2 v_{0}+\frac{3}{4\left(\lambda^{2}-1\right)}\right] \mathrm{g}\right\} \\
& \left.\mathrm{S}_{1122}=\mathrm{S}_{1133}=-\frac{1}{\lambda^{2}}+\frac{1}{2\left(1-v_{0}\right)}\left[1-2 v_{0}+\frac{1}{\lambda^{2}-1}\right]+\frac{3 \lambda^{2}}{2\left(1-v_{0}\right)}-\left(1-2 v_{0}\right)\right] \mathrm{g} \\
& \mathrm{S}_{2323}=\frac{1}{4\left(1-v_{0}\right)}\left[1-2 v_{0}+\frac{3}{\lambda^{2}-1}\right] \mathrm{g} \\
& \left.\mathrm{S}_{1212}=\mathrm{S}_{1313}=\frac{1}{2\left(\lambda^{2}-1\right)}+\left[1-2 v_{0}-\frac{3}{4\left(\lambda^{2}-1\right)}\right] \mathrm{g}\right\}
\end{aligned}
$$

where $\nu_{0}$ is Poisson's ratio of the matrix, $\lambda$ is the aspect ratio of the inclusion and $\mathrm{g}$ is given by

$$
g= \begin{cases}\frac{\lambda}{\left(\lambda^{2}-1\right)^{3 / 2}}\left\{\lambda\left(\lambda^{2}-1\right)^{1 / 2}-\arccos \lambda\right\}, \quad \lambda>1 \\ \frac{\lambda}{\left(\lambda^{2}-1\right)^{3 / 2}}\left\{\arccos \lambda-\lambda\left(1-\lambda^{2}\right)^{1 / 2}\right\}, \quad \lambda<1\end{cases}
$$

For a spherical inclusion, they simplify to

$$
\begin{aligned}
& \mathrm{S}_{1111}=\mathrm{S}_{2222}=\mathrm{S}_{3333}=\frac{7-5 v_{0}}{15\left(1-v_{0}\right)} \\
& \mathrm{S}_{1122}=\mathrm{S}_{2233}=\mathrm{S}_{3311}=\frac{5 v_{0}-1}{15\left(1-v_{0}\right)} \\
& \mathrm{S}_{1212}=\mathrm{S}_{2323}=\mathrm{S}_{3131}=\frac{4-5 v_{0}}{15\left(1-v_{0}\right)}
\end{aligned}
$$




\section{Advances in Engineering Plasticity}

10.4028/www.scientific.net/KEM.177-180

\section{Embedded Cell Model of Three-Dimensional Two-Phase Composites}

10.4028/www.scientific.net/KEM.177-180.291

\section{DOI References}

[4] B. Budiansky, I Mech. Phys. Solids, 13 (1965) 223-227.

doi:10.1016/0022-5096(65)90011-6

[6] R. Mclaughlin, mt. I EngngSci., 15 (1977) 237-244.

doi:10.1016/0020-7225(77)90058-1

[9] Q. S. Zheng and D. X. Du, Key Engineering Materials, 145-149 (1998) 479-488.

doi:10.4028/www.scientific.net/KEM.145-149.479

[11] J. M. Duva and J. W. Hutchinson, Mech. Mater., 3 (1984) 41-54.

doi:10.1016/0167-6636(84)90013-9

[13] J. Llorca and C. Gonzalez, I Mech. Phys. Solids, 40 (1998) 1-28.

doi:10.1023/A:1021738921514

[15] K. Tanaka and T. Mori, I Elasticity, 2 (1972) 199-200.

doi:10.1007/BF00125528 